\title{
Trading Strategies for Distribution Company with Stochastic Distributed Energy Resources
}

\author{
Chunyu Zhang ${ }^{\mathrm{a}}$, Qi Wang ${ }^{\mathrm{b}}$, Jianhui Wang, ${ }^{\mathrm{c}, *}$, Magnus Korpås ${ }^{\mathrm{a}}$, Pierre \\ Pinson $^{\mathrm{b}}$, Jacob Østergaard ${ }^{\mathrm{b}}$, Mohammad E. Khodayar $^{\mathrm{d}}$ \\ ${ }^{a}$ Department of Electric Power Engineering, Norwegian University of Science and \\ Technology, Trondheim, Norway \\ ${ }^{b}$ Center for Electric Power and Energy, Technical University of Denmark, Lyngby, \\ Denmark \\ ${ }^{c}$ Energy Systems Division, Argonne National Laboratory, Argonne, IL, USA \\ ${ }^{d}$ Electrical Engineering Department, Southern Methodist University, Dallas, TX, USA
}

\begin{abstract}
This paper proposes a methodology to address the trading strategies of a proactive distribution company (PDISCO) engaged in the transmission-level (TL) markets. A one-leader multi-follower bilevel model is presented to formulate the gaming framework between the PDISCO and markets. The lower-level (LL) problems include the TL day-ahead market and scenario-based real-time markets, respectively with the objectives of maximizing social welfare and minimizing operation cost. The upper-level (UL) problem is to maximize the PDISCO's profit across these markets. The PDISCO's strategic offers/bids interactively influence the outcomes of each market. Since the LL problems are linear and convex, while the UL problem is non-linear and non-convex, an equivalent primal-dual approach is used to reformulate this bilevel model to a solvable mathematical program with equilibrium constraints (MPEC). The effectiveness of the proposed model is verified by case studies.
\end{abstract}

Keywords: Distributed energy resources (DERs), proactive distribution company (PDISCO), electricity markets, bilevel game-theoretic model,

\footnotetext{
${ }^{*}$ Corresponding author

Email addresses: chunyu.zhang@ntnu.no (Chunyu Zhang), qiwa@elektro.dtu.dk (Qi Wang), jianhui.wang@anl.gov (Jianhui Wang), magnus.korpas@ntnu.no (Magnus Korpås), ppin@elektro.dtu.dk (Pierre Pinson), joe@elektro.dtu.dk (Jacob Østergaard), mkhodayar@smu.edu (Mohammad E. Khodayar)
}

Preprint submitted to Applied Energy

May 24, 2016

(C) 2016. This manuscript version is made available under the Elsevier user license http://www.elsevier.com/open-access/userlicense/1.0/ 
multi-period AC power flow, mathematical program with equilibrium constraints (MPEC), mathematical program with primal and dual constraints (MPPDC).

\section{Nomenclature}

Sets and Indices

$i, j, B^{D S}$
$n, m, B^{T S}$$\quad$ Index and set of distribution-level (DL) and transmission-level (TL) buses, respectively.

$5 \quad i j, \Lambda^{D S}$

$l, L$

$d, D$

$g, G$

$k, K$

$t, T$

$10 \omega, \Omega$

$\mathcal{M}_{L}, \mathcal{M}_{D}$

$\mathcal{M}_{G}$

15

$\mathcal{M}_{K}$

Variables

$P_{t g}^{G}$

$R_{t g}^{U P}, R_{t g}^{D N}$

${ }_{20} r_{t g \omega}^{U P}, r_{t g \omega}^{D N}$
Index and set of DL feeders and TL lines, respectively.

Index and set of DL and TL demands, respectively.

Index and set of TL generators.

Index and set of DL DERs.

Index and set of time periods (e.g., hours per day).

Index and set of scenarios.

Mapping of the set of DL/TL demands onto the set of DL/TL buses respectively.

Mapping of the set of TL conventional generations onto the set of TL buses.

Mapping of the set of DERs onto the set of DL buses.

Day-ahead offer of generator $g$ at time $t$.

Day-ahead up and down regulation reserve capacities of generator $g$ at time $t$.

Real-time up and down regulation power of generator $g$ at time $t$ for scenario $\omega$. 
$\lambda_{t}^{D D A} \quad$ Day-ahead offering/bidding price of the PDISCO at time $t$.

$P_{t}^{D D A}, Q_{t}^{D D A} \quad$ Day-ahead offering/bidding quantity of the PDISCO at time $t$ (non-negative is offer, negative is bid).

${ }_{25} \lambda_{t \omega}^{D R T}$

Real-time offering/bidding price of the PDISCO at time $t$ for scenario $\omega$.

$P_{t \omega}^{D R T}, Q_{t \omega}^{D R T} \quad$ Real-time offering/bidding quantity of the PDISCO at time $t$ for scenario $\omega$ (non-negative is offer, negative is bid).

$P_{t d \omega}^{S T} \quad$ TL load-shedding of demand $d$ at time $t$ for scenario $\omega$.

30 $\theta_{t n}^{0}, \theta_{t n \omega} \quad$ Voltage angles of bus $n$ at day-ahead time $t$, and at real-time time $t$ for scenario $\omega$.

$\lambda_{t n}^{D A}, \lambda_{t n \omega}^{R T} \quad$ Locational marginal price (LMP) at TL bus $n$ at day-ahead time $t$, and at real-time time $t$ for scenario $\omega$.

$P_{t k}^{D E R 0}, Q_{t k}^{D E R 0}$ Active and reactive power procured from DER $k$ at day-ahead $P_{t, i j, \omega}^{F D}, Q_{t, i j, \omega}^{F D}$ $\delta_{t i}^{0}, \delta_{t i \omega}$ $V_{t i}^{0}, V_{t i \omega}$ time $t$.

Active and reactive power of DL load-shedding for demand $l$ at time $t$ for scenario $\omega$.

Reactive power from DL shunt compensator at bus $i$ at dayahead time $t$, and at real-time time $t$ for scenario $\omega$.

Day-ahead active and reactive power flows through DL feeder $i-j$ at time $t$.

Real-time active and reactive power flows through DL feeder $i$ - $j$ at time $t$ for scenario $\omega$.

Voltage angles of bus $i$ at day-ahead time $t$, and at real-time time $t$ for scenario $\omega$.

Voltage magnitudes of DL bus $i$ at day-ahead time $t$, and at real-time time $t$ for scenario $\omega$. 
Parameters

$P_{t k \omega}^{D E R}, Q_{t k \omega}^{D E R} \quad$ Active and reactive power generation realization of DER $k$ at time $t$ for scenario $\omega$.

$\bar{P}_{g}^{G}, \bar{R}_{g}^{U P}, \bar{R}_{g}^{D N}$ Maximum production, maximum up and down regulation reserve capacities of generator $g$.

$C_{g}^{G}, C_{g}^{U P}, C_{g}^{D N}$ Day-ahead generation cost, up and down regulation reserve costs of generator $g$.

${ }_{55} c_{g}^{U P}, c_{g}^{D N}$ Real-time up and down regulation cost of generator $g$.

$C_{t}^{D S}$ Operation cost of the PDISCO at time $t$.

$\lambda_{t d}^{T S D}, P_{t d}^{T S D} \quad$ Day-ahead bidding price and consumption of TL demand $d$ at time $t$.

$\lambda_{t}^{D E R} \quad$ DER procurement price of the PDISCO at time $t$.

${ }_{60} \quad \lambda_{t}^{D S D}$ DL sale price at time $t$.

$P_{t l}^{D S D}, Q_{t l}^{D S D} \quad$ Consumption of DL demand $l$ at time $t$.

$\bar{P}^{D S} \quad$ Active power injection limit for the PDISCO.

$\lambda_{t}^{S T}, \lambda_{t}^{S D} \quad$ TL/DL load-shedding price at time $t$.

$\bar{P}_{n m}^{T S} \quad$ Capacity limit of each TL line $n m$.

${ }_{65} \bar{S}, \bar{S}_{i j} \quad$ Capacity limits of the DL main substation and each DL feeder $i j$.

$\bar{S}_{k} \quad$ Capacity limit of each DER $k$

$\underline{Q}_{i}^{C}, \bar{Q}_{i}^{C} \quad$ Reactive power limits of the DL shunt compensator at bus $i$.

$\underline{V}_{i}, \bar{V}_{i} \quad$ Limits of voltage magnitude at DL bus $i$.

${ }_{70} \tau_{i}$

Transformer tap ratio at DL bus $i$. 
$B_{n m} \quad$ Susceptance of the TL line $n m$.

$G_{i j}, B_{i j}, b_{i j} \quad$ Conductance, susceptance and charging susceptance of the DL feeder $i j$.

\section{Introduction}

Distributed energy resources (DERs) tend to occupy a high share in the distribution-level (DL) network [1, 2]. In a deregulated environment, this stimulates the distribution company (DISCO) to preferentially procure DERs' generations at low prices. In the U.S., the recent initiative named the New York Reforming Energy Vision (NY REV) [3] has addressed the regulatory changes

so to liberate a DL market for cost-effective use of DERs. As indicated in the NY REV, a Distributed System Platform Provider (DSPP) will modernize its distribution system to create a flexible platform for new energy products and services, to improve the overall system efficiency. Resources provided could include distributed generation (DG), energy efficiency, predictive demand management, demand response (DR), microgrids (MGs), and more. This paper is partially motivated by the NY REV and aims to establish a real-time market framework for the PDISCO procuring the DL DERs and trading in the transmission-level (TL) wholesale markets. The DL resources are selected as stochastic DERs, such as wind turbines (WTs) and photovoltaic systems (PVs), while the proactive DISCO (PDISCO) can be considered as a DSPP to play an essential role in the hierarchical trading framework. To this end, the PDISCO gets an opportunity to strategically engage in the TL markets by rationally purchasing electricity from the DL DERs. In this situation, the DISCO's trading framework becomes more complex.

95 Associated with the smart grid technology, to participate in the day-ahead and real-time markets, for each time $t$, the DISCO has to make a trade-off on acquiring DERs' portfolio and trading strategy (offer/bid) to maximize its profit. Crossing the two-stage markets, the transactions between the DISCO and markets are characterized in a bidirectional fashion, implying the DISCO 
behaves as an active producer when providing offers, but as an active consumer when submitting bids. To highlight these features, this kind of DISCO is defined as a PDISCO in this paper. On the other hand, the PDISCO's trading strategies (offering/bidding prices and power quantities) are endogenously interrelated with the markets' outcomes (locational marginal prices (LMPs) and production/consumption quantities). Thus, the trading between the PDISCO and markets follows a typical gaming structure.

In order to capture the PDISCO's trading strategies, the PDISCO trading within markets can be formulated as a one-leader multi-follower game model, realized in a bilevel structure. Market-clearing procedures are indicated as the stage-based LL problems through DC power flow. The LL day-ahead market problem is to maximize the TL social welfare. In particular, in the real-time process, scenario-based methods [4] can be used to represent the stochastic outputs of individual DERs. Accordingly, an LL real-time market problem seeks to minimize the TL operation cost per scenario $\omega$. The UL problem represents the profit maximization of the PDISCO, with the strategic offers/bids constrained by $\mathrm{AC}$ power flow. Note that the LL problems are linear and convex, while the UL problem is non-linear and non-convex. The primal-dual approach [5] is applied to reformulate the proposed bilevel model to a solvable mathematical program with equilibrium constraints (MPEC).

Few papers are available in the technical literature to discuss the DISCO trading within the TL markets. A static bilevel model is proposed in [6] to support a DISCO's operational decision with DGs and interruptible loads (ILs) in a competitive market, while the DISCO's offers/bids are ignored by the market objective. To optimize the DISCO's day-ahead acquisition, a static bilevel model [7] is presented to purchase energy from the wholesale market, ILs and dispatchable DGs, neglecting their stochastic outputs. For the same purpose, a multi-period bilevel model is further presented in [8] to exhibit the competitiveness of ILs and the DISCO-owned DGs, but the model excludes the network constraints. To address a proposed DL market framework by interacting with the TL markets, assuming the prices from TL markets are known as parame- 
ters, the PDISCO procurement strategies with the DL aggregator-based DRs [9] and stochastic DGs [10] are fully presented and discussed by one-leader multifollower bilevel models, respectively.

At differing market stages, bilevel modeling is increasingly used to determine the trading strategies [11]. For instance, in [12], given wind power production as a set of correlated scenarios, a scenario-based bilevel model is presented to derive strategic offers for a wind producer within markets. To minimize the payment in pool markets, a multi-period bilevel model [13] is proposed to address the consumers' strategies in terms of LMPs. From procurement perspectives, to establish a robust retail market, the role of a utility is suggested as a portfolio manager [14. For a community-scale MG, a two-stage stochastic program is used to optimize its power scheduling and bidding strategy on the basis of dayahead prices [15].

Regarding the energy development and policy, the renewable energy procurement and the related incentive policies in the state of Illinois in the U.S. are reviewed and discussed in [16. The economic value of wind-based DGs is addressed in [17] by applying a Longstaff-Schwartz option pricing method. To evaluate the impact of residential DR choices considering uncertainties, a probabilistic methodology is presented to trade DR in a distribution energy market 150 [18. In competitive energy markets, the benefits of the Community Energy Storage (CES) devices are analyzed in [19] for optimizing the power system operations. Storage-like devices are also investegated in load leveling 20] through complementarity constraints and a new and exact relaxation method. In [21, new functionalities of utilities and distributed electricity prosumers are indentified and cleared with the retail electricity market price. In order to faciliate large-scale EV charging facilities and energy storage devices participating in frequency regulation, 22 proposes control strategies to enhance the integration of renewable energy.

In view of the context above, the contributions of this paper are threefold:

1) Present a framework for the PDISCO trading in the day-ahead and realtime markets. 
2) Propose a bilevel model for the PDISCO to derive continuous strategic offers and bids by rationally procuring the DERs' portfolio.

3) Reformulate the PDISCO's game-theoretic model to an MPEC by replacing the LL market problems with an equivalent primal-dual approach.

The rest of this paper is organized as follows. Section 2 presents the framework of the PDISCO trading in TL markets and procuring from DL DERs. The corresponding bilevel game-theoretic model is proposed in Section 3, in which the primal-dual approach is also indicated in detail. In Section 4 , the numerical results of the case studies are reported and analyzed. Finally, some relevant remarks are concluded in Section 5.

\section{PDISCO Trading in Markets}

Till now, at the transmission level, electricity markets are normally recognized as wholesale markets and realized as pools. For short-term transactions, the electricity can be traded in the day-ahead and real-time markets. Each wholesale market follows a auction framework, which is commonly applied to find a trade-off between the offers and bids submitted by producers and consumers, respectively. Each offer/bid is specified as a set of price-quantity pairs. On the other hand, if the transmission network is considered in a market-clearing procedure, a LMP occurs at each bus, covering line congestion and line losses [5.

As shown in Fig.1, to trade in the TL markets, the PDISCO has two optional strategies to take part in each market, i.e., offer or bid in the day-ahead market and real-time market per scenario $\omega$. For two-stage market operation, the dayahead market is cleared prior to the real-time markets. Therefore, at each time $t$, the PDISCO's strategic day-ahead offer/bid $\left(\lambda_{t}^{D D A}, P_{t}^{D D A}\right)$ goes with the dayahead market aiming to meet the TL demands by generators' offers, while each real-time market balances the PDISCO's strategic real-time offer/bid $\left(\lambda_{t \omega}^{D R T}\right.$, $P_{t \omega}^{D R T}$ ) with the TL regulation power and/or load-shedding.

Crossing the markets, to maximize profit, the PDISCO's power quantity 


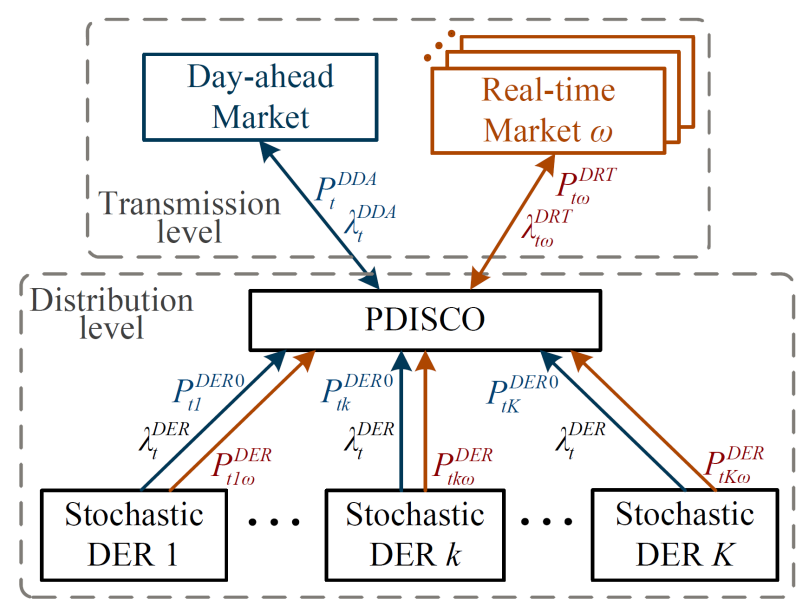

Figure 1: PDISCO trading in markets.

$P_{t}^{D D A}$ is scheduled by rationally procuring the DERs' portfolios (a set of $P_{t k}^{D E R 0}$ ) against physical DL constraints at the day-ahead stage, and the scenario-based power quantity $P_{t \omega}^{D R T}$ is determined as the variation pertaining to the total DERs' outputs $\left(\sum_{k} P_{t k \omega}^{D E R}\right)$ versus network constraints at the real-time stage. At each time $t$ : If $P_{t}^{D D A} / P_{t \omega}^{D R T}$ is non-negative, the PDISCO behaves as an active producer with a lower offering price $\lambda_{t}^{D D A} / \lambda_{t \omega}^{D R T}$; If this quantity is negative, the PDISCO acts as an active consumer with a higher bidding price. For the stochastic DERs, the PDISCO's procurements are settled with the price $\lambda_{t}^{D E R}$, and the scenario-based method [4] can be used to represent the individual DERs' uncertainties.

\section{Problem Formulation}

Participating in the TL markets, the PDISCO's strategic trading problem can be formulated as a bilevel one-leader multi-follower game-theoretic model 5. One follower is characterized as an LL day-ahead market problem with the goal to maximize the TL social welfare. Other followers are scenario-based LL real-time market problems. An LL problem per scenario $\omega$ seeks to achieve the cost minimization during the real-time TL balancing. In particular, to 
obtain the trade-offs between the markets, the offering/bidding strategies of the PDISCO are conducted by the UL problem with the purpose of minus-profit minimization.

\subsection{Assumptions}

The assumptions of the proposed bilevel model are as follows:

1) The day-ahead and real-time offering/bidding strategies of a single PDISCO are considered to be involved in the corresponding TL markets.

2) The DL network is assumed to be operated and owned by the PDISCO, and interconnected to the TL network via only one main substation.

3) Only the active power is considered to be traded in markets. At each time $t$, we assume the reactive power exchanging between DL and TL can be balanced at the DL main substation.

4) The PDISCO can explicitly anticipate the impacts of its strategic offers/bids, versus the markets' outcomes. One offer/bid per time $t$ for each market.

\subsection{LL problems}

Note that the outcomes of the LL problems can directly affect the PDISCO's offering/bidding strategies in the UL problem. Thus, the corresponding LL problem formulations are given below.

1) LL day-ahead market problem:

$$
\begin{aligned}
& \underset{\Xi^{D A T}}{\operatorname{Minimize}} \sum_{t, g}\left(C_{g}^{G} P_{t g}^{G}+C_{g}^{U P} R_{t g}^{U P}+C_{g}^{D N} R_{t g}^{D N}\right) \\
& +\sum_{t} \lambda_{t}^{D D A} P_{t}^{D D A}-\sum_{t, d} \lambda_{t d}^{T S D} P_{t d}^{T S D}
\end{aligned}
$$

s.t.

For PDISCO's location bus $I$ :

$$
\sum_{(g: I) \in \mathcal{M}_{G}} P_{t g}^{G}+P_{t}^{D D A}=\sum_{I m \in \Lambda^{T S}} B_{I m}\left(\theta_{t I}^{0}-\theta_{t m}^{0}\right): \lambda_{t I}^{D A}
$$$$
, \forall t
$$ 


$$
-\bar{P}^{D S} \leq P_{t}^{D D A} \leq \bar{P}^{D S}: \beta_{t}^{0-}, \beta_{t}^{0+}, \forall t
$$

For other buses:

$$
\begin{aligned}
& \sum_{(g: n) \in \mathcal{M}_{G}} P_{t g}^{G}-P_{t d}^{T S D}=\sum_{n m \in \Lambda^{T S}} B_{n m}\left(\theta_{t n}^{0}-\theta_{t m}^{0}\right) \\
& : \lambda_{t n}^{D A}, \forall t, n_{(d: n) \in \mathcal{M}_{D}} \\
& 0 \leq P_{t g}^{G}+R_{t g}^{U P} \leq \bar{P}_{t g}^{G}: \gamma_{t g}^{0-}, \gamma_{t g}^{0+}, \forall t, g \\
& 0 \leq P_{t g}^{G}-R_{t g}^{D N}: \eta_{t g}^{0}, \forall t, g \\
& 0 \leq R_{t g}^{U P} \leq \bar{R}_{g}^{U P}: \phi_{t g}^{0-}, \phi_{t g}^{0+}, \forall t, g \\
& 0 \leq R_{t g}^{D N} \leq \bar{R}_{g}^{D N}: \psi_{t g}^{0-}, \psi_{t g}^{0+}, \forall t, g \\
& -\bar{P}_{n m}^{T S} \leq B_{n m}\left(\theta_{t n}^{0}-\theta_{t m}^{0}\right) \leq \bar{P}_{n m}^{T S}: \mu_{t, n m}^{0-}, \mu_{t, n m}^{0+}, \\
& \forall t, n m \in \Lambda^{T S} \\
& -\pi \leq \theta_{t n}^{0} \leq \pi: \sigma_{t n}^{0-}, \sigma_{t n}^{0+}, \forall t, n \\
& \theta_{t 1}^{0}=0: \zeta_{t}^{0}, \forall t, n=1
\end{aligned}
$$

where $\Xi^{D A T}=\left\{P_{t g}^{G}, R_{t g}^{U P}, R_{t g}^{D N}, P_{t}^{D D A}, \theta_{t n}^{0}\right\}$ is the variable set of the LL dayahead problem.

The day-ahead market clearing is modeled within the LL problem (1). The objective function (1.1) is to minimize the minus (maximize) TL social welfare, i.e., the generation and reserve cost of individual generators plus the PDISCO's strategic offers/bids (non-negative $P_{t}^{D D A}$ is offer, while the negative is bid, $\forall t$ ), and minus the revenue of sales to the other TL demands. We assume all the generators are dispatchable. DC power flow is adopted to formulate the TL operation conditions. At each time $t$ : Constraints (1.2) and (1.4) represent the power balance at the PDISCO's location bus $I$ and the other TL buses, respectively. Constraints (1.3) impose the bounds of the PDISCO's offering/bidding quantity $P_{t}^{D D A}$. Constraints (1.5) (1.6) (1.7) and (1.8) specify the capacity limits of the up/down reserve $R_{t g}^{U P} / R_{t g}^{D N}$ for generator $g$. Constraints (1.9) indicate 240 the capacity limits of the TL line $n$ - $m$. Constraints (1.10) express the range of the TL voltage angles, and constraints (1.11) set TL bus 1 as the reference bus. Correspondingly, the dual variables for each group of constraints are indicated 
at the right side of a colon.

2) LL real-time market problems:

$$
\begin{aligned}
& \left\{\underset{\Xi R T T}{\operatorname{Minimize}} \sum_{t, g}\left(c_{g}^{U P} r_{t g \omega}^{U P}-c_{g}^{D N} r_{t g \omega}^{D N}\right)\right. \\
& +\sum_{t} \lambda_{t \omega}^{D R T} P_{t \omega}^{D R T}+\sum_{t, d} \lambda_{t}^{S T} P_{t d \omega}^{S T}
\end{aligned}
$$

s.t.

For PDISCO's location bus $I$ :

$$
\begin{aligned}
& \sum_{(g: I) \in \mathcal{M}_{G}}\left(r_{t g \omega}^{U P}-r_{t g \omega}^{D N}\right)+P_{t \omega}^{D R T}=\sum_{I m \in \Lambda^{T S}} B_{I m} \\
& \left(\theta_{t I \omega}-\theta_{t I}^{0}-\theta_{t m \omega}+\theta_{t m}^{0}\right): \lambda_{t I \omega}^{R T}, \forall t \\
& -\bar{P}^{D S} \leq P_{t \omega}^{D R T} \leq \bar{P}^{D S}: \beta_{t \omega}^{-}, \beta_{t \omega}^{+}, \forall t
\end{aligned}
$$

For other buses:

$$
\begin{aligned}
& \sum_{(g: I) \in \mathcal{M}_{G}}\left(r_{t g \omega}^{U P}-r_{t g \omega}^{D N}\right)+P_{t d \omega}^{S T}=\sum_{n m \in \Lambda^{T S}} B_{n m} \\
& \left(\theta_{t n \omega}-\theta_{t n}^{0}-\theta_{t m \omega}+\theta_{t m}^{0}\right): \lambda_{t n \omega}^{R T}, \forall t, n_{(d: n) \in \mathcal{M}_{D}} \\
& 0 \leq r_{t g \omega}^{U P} \leq R_{t g}^{U P}: \phi_{t g \omega}^{-}, \phi_{t g \omega}^{+}, \forall t, g \\
& 0 \leq r_{t g \omega}^{D N} \leq R_{t g}^{D N}: \psi_{t g \omega}^{-}, \psi_{t g \omega}^{+}, \forall t, g \\
& 0 \leq P_{t d \omega}^{S T} \leq P_{t d}^{T S D}: \nu_{t d \omega}^{-}, \nu_{t d \omega}^{+}, \forall t, d \\
& -\bar{P}_{n m}^{T S} \leq B_{n m}\left(\theta_{t n \omega}-\theta_{t m \omega}\right) \leq \bar{P}_{n m}^{T S}: \mu_{t, n m, \omega}^{-}, \\
& \mu_{t, n m, \omega}^{+}, \forall t, n m \in \Lambda^{T S} \\
& -\pi \leq \theta_{t n \omega} \leq \pi: \sigma_{t n \omega}^{-}, \sigma_{t n \omega}^{+}, \forall t, n \\
& \left.\theta_{t 1 \omega}=0: \zeta_{t \omega}, \forall t, n=1\right\} \forall \omega .
\end{aligned}
$$

where $\Xi^{R T T}=\left\{r_{t g \omega}^{U P}, r_{t g \omega}^{D N}, P_{t \omega}^{D R T}, P_{t d \omega}^{S T}, \theta_{t n \omega}\right\}$ is the variable set of each LL real-time problem per scenario $\omega$. At this stage, the day-ahead market variables $R_{t g}^{U P}, R_{t g}^{D N}$ and $\theta_{t n}^{0}$ are recognized as parameters.

The real-time market is cleared by (2). For each scenario $\omega$, the objective (2.1) indicates the TL operation cost minimization, which comprises the regula- 
tion cost of each generator $g$, the strategic offers/bids of the PDISCO, and the cost of load-shedding invocations. On the basis of DC power flow, at each time $t$ per scenario $\omega$ : Constraints (2.2) guarantee the power balance at the PDISCO bus $I$ to deal with its strategic offering/bidding quantity $P_{t \omega}^{D R T}$, which is further limited in (2.3). For other buses, constraints (2.4) ensure the power balance at each bus when involuntary load-shedding occurs. Constraints (2.5) and (2.6) enforce the up and down regulation $\left(r_{t g \omega}^{U P}, r_{t g \omega}^{D N}\right)$ of each generator should not exceed its up and down reserve capacity $\left(R_{t g}^{U P}, R_{t g}^{D N}\right)$, respectively. Constraints (2.7) indicate the TL load-shedding limits. Constraints (2.8) identify the transmission capacity bounds. Constraints (2.9) and (2.10) limit the real-time TL voltage angle at bus $n$. The related dual variables are also separated by a colon.

\subsection{UL problem}

Interrelated with the LL problems, the offers/bids determined by the UL problem inevitably influence the markets' outcomes in the LL problems. Therefore, the UL problem covers the PDISCO's offering/bidding strategies in individual markets, DL network constraints, and markets' arguments regarding the LMPs and production/consumption quantities.

$$
\begin{aligned}
& \underset{\substack{\bar{D}^{D A T} \cup \Xi^{R T T} \\
\cup \Xi D I S C O \cup \Xi D \text { ual }}}{\operatorname{Minimize}}-\sum_{t}\left(\lambda_{t I}^{D A}-C_{t}^{D S}\right) P_{t}^{D D A} \\
& +\sum_{t, k} \lambda_{t}^{D E R} P_{t k}^{D E R 0}-\sum_{t, l} \lambda_{t}^{D S D} P_{t l}^{D S D}+ \\
& \mathbb{E}\left[-\sum_{t}\left(\lambda_{t I \omega}^{R T}-C_{t}^{D S}\right) P_{t \omega}^{D R T}+\sum_{t, l}\left(\lambda_{t}^{S D}+\lambda_{t}^{D S D}\right) P_{t l \omega}^{S D}\right. \\
& \left.-\sum_{t, k}\left(\lambda_{t I \omega}^{R T}-\lambda_{t}^{D E R}\right)\left(P_{t k \omega}^{D E R}-P_{t k}^{D E R 0}\right)\right]
\end{aligned}
$$

PDISCO day-ahead constraints (3.2)-(3.16):

$$
P_{t}^{D D A} \leq \sum_{k} P_{t k}^{D E R 0}-\sum_{l} P_{t l}^{D S D}, \forall t
$$

For the main substation (PDISCO's reference bus 1): 


$$
\begin{aligned}
& -P_{t}^{D D A}+\sum_{(k: 1) \in \mathcal{M}_{K}} P_{t k}^{D E R 0}-P_{t 1}^{D S D}=\sum_{1 j \in \Lambda^{D S}} P_{t, 1 j}^{F D 0}, \forall t \\
& -Q_{t}^{D D A}+\sum_{(k: 1) \in \mathcal{M}_{K}} Q_{t k}^{D E R 0}-Q_{t 1}^{D S D}+Q_{t 1}^{C 0} \\
& =\sum_{1 j \in \Lambda^{D S}} Q_{t, 1 j}^{F D 0}, \forall t \\
& V_{t 1}^{0}=1, \forall t \\
& \delta_{t 1}^{0}=0, \forall t \\
& \left(P_{t}^{D D A}\right)^{2}+\left(Q_{t}^{D D A}\right)^{2} \leq \bar{S}, \forall t
\end{aligned}
$$

For the other buses:

$$
\begin{aligned}
& \sum_{(k: i) \in \mathcal{M}_{K}} P_{t k}^{D E R 0}-P_{t l}^{D S D}=\sum_{i j \in \Lambda^{D S}} P_{t, i j}^{F D 0}, \forall t, i_{(l: i) \in \mathcal{M}_{L}} \\
& \sum_{(k: i) \in \mathcal{M}_{K}} Q_{t k}^{D E R 0}-Q_{t l}^{D S D}+Q_{t i}^{C 0}=\sum_{i j \in \Lambda^{D S}} Q_{t, i j}^{F D 0}, \\
& \forall t, i_{(l: i) \in \mathcal{M}_{L}} \\
& P_{t, i j}^{F D}=-\tau_{i}\left(V_{t i}^{0}\right)^{2} G_{i j}+V_{t i}^{0} V_{t j}^{0}\left[G_{i j} \cos \left(\delta_{t i}^{0}-\delta_{t j}^{0}\right)\right. \\
& \left.+B_{i j} \sin \left(\delta_{t i}^{0}-\delta_{t j}^{0}\right)\right], \forall t, i j \in \Lambda^{D S} \\
& Q_{t, i j}^{F D 0}=\tau_{i}\left(V_{t i}^{0}\right)^{2} B_{i j}-0.5 b_{i j}+V_{t i}^{0} V_{t j}^{0}\left[G_{i j} \sin \left(\delta_{t i}^{0}-\delta_{t j}^{0}\right)\right. \\
& \left.-B_{i j} \cos \left(\delta_{t i}^{0}-\delta_{t j}^{0}\right)\right], \forall t, i j \in \Lambda^{D S} \\
& \left(P_{t, i j}^{F D 0}\right)^{2}+\left(Q_{t, i j}^{F D 0}\right)^{2} \leq\left(\bar{S}_{i j}\right)^{2}, \forall t, i j \in \Lambda^{D S} \\
& \left(P_{t k}^{D E R 0}\right)^{2}+\left(Q_{t k}^{D E R 0}\right)^{2} \leq\left(\bar{S}_{k}\right)^{2}, \forall t, k \\
& \underline{Q}_{i}^{C} \leq Q_{t i}^{C 0} \leq \bar{Q}_{i}^{C}, \forall t, i \\
& \underline{V_{i} \leq V_{t i}^{0} \leq \bar{V}_{i}, \forall t, i} \\
& -\pi \leq \delta_{t i}^{0} \leq \pi, \forall t, i
\end{aligned}
$$

PDISCO real-time constraints (3.17)-(3.33):

$$
P_{t \omega}^{D R T} \leq \sum_{k}\left(P_{t k \omega}^{D E R}-P_{t k}^{D E R 0}\right)+\sum_{l} P_{t l \omega}^{S D}, \forall t, \omega
$$

For the main substation (PDISCO's reference bus 1): 


$$
\begin{aligned}
& -P_{t \omega}^{D R T}+\sum_{(k: 1) \in \mathcal{M}_{K}}\left(P_{t k \omega}^{D E R}-P_{t k}^{D E R 0}\right)+P_{t 1 \omega}^{S D} \\
& =\sum_{1 j \in \Lambda^{D S}}\left(P_{t, 1 j \omega}^{F D}-P_{t, 1 j}^{F D 0}\right), \forall t, \omega \\
& -Q_{t \omega}^{D R T}+\sum_{(k: 1) \in \mathcal{M}_{K}}\left(Q_{t k \omega}^{D E R}-Q_{t k}^{D E R 0}\right)+Q_{t 1 \omega}^{S D}+Q_{t 1 \omega}^{C} \\
& -Q_{t 1}^{C 0}=\sum_{1 j \in \Lambda^{D S}}\left(Q_{t, 1 j \omega}^{F D}-Q_{t, 1 j}^{F D 0}\right), \forall t, \omega \\
& V_{t 1 \omega}=1, \forall t, \omega \\
& \delta_{t 1 \omega}=0, \forall t, \omega \\
& \left(P_{t}^{D D A}+P_{t \omega}^{D R T}\right)^{2}+\left(Q_{t}^{D D A}+Q_{t \omega}^{D R T}\right)^{2} \leq \bar{S}^{2}, \forall t, \omega
\end{aligned}
$$

For the other buses:

$$
\begin{aligned}
& \sum_{(k: i) \in \mathcal{M}_{K}}\left(P_{t k \omega}^{D E R}-P_{t k}^{D E R 0}\right)+P_{t l \omega}^{S D} \\
& =\sum_{i j \in \Lambda^{D S}}\left(P_{t, i j, \omega}^{F D}-P_{t, i j}^{F D 0}\right), \forall t, i_{(l: i) \in \mathcal{M}_{L}}, \omega \\
& \sum_{(k: i) \in \mathcal{M}_{K}}\left(Q_{t k \omega}^{D E R}-Q_{t k}^{D E R 0}\right)+Q_{t l \omega}^{S D}+Q_{t i \omega}^{C}-Q_{t i}^{C 0} \\
& =\sum_{i j \in \Lambda^{D S}}\left(Q_{t, i j, \omega}^{F D}-Q_{t, i j}^{F D 0}\right), \forall t, i_{(l: i) \in \mathcal{M}_{L}}, \omega \\
& P_{t, i j, \omega}^{F D}=-\tau_{i} V_{t i \omega}^{2} G_{i j}+V_{t i \omega} V_{t j \omega}\left[G_{i j} \cos \left(\delta_{t i \omega}-\delta_{t j \omega}\right)\right. \\
& \left.+B_{i j} \sin \left(\delta_{t i \omega}-\delta_{t j \omega}\right)\right], \forall t, i j \in \Lambda^{D S}, \omega \\
& Q_{t, i j, \omega}^{F D}=\tau_{i} V_{t i \omega}^{2} B_{i j}-0.5 b_{i j}+V_{t i \omega} V_{t j \omega}\left[G_{i j} \sin \left(\delta_{t i \omega}-\delta_{t j \omega}\right)\right. \\
& \left.-B_{i j} \cos \left(\delta_{t i \omega}-\delta_{t j \omega}\right)\right], \forall t, i j \in \Lambda^{D S}, \omega \\
& \left(P_{t, i j, \omega}^{F D}\right)^{2}+\left(Q_{t, i j, \omega}^{F D}\right)^{2} \leq\left(\bar{S}_{i j}\right)^{2}, \forall t, i j \in \Lambda^{D S}, \omega \\
& 0 \leq P_{t l \omega}^{S D} \leq P_{t l}^{D S D}, \forall t, l, \omega \\
& 0 \leq Q_{t l \omega}^{S D} \leq Q_{t l}^{D S D}, \forall t, l, \omega \\
& P_{t l}^{D S D} Q_{t l \omega}^{S D}-P_{t l \omega}^{S D} Q_{t l}^{D S D}=0, \forall t, l, \omega \\
& \underline{Q}_{i}^{C} \leq Q_{t i \omega}^{C} \leq \bar{Q}_{i}^{C}, \forall t, i, \omega \\
& \underline{V}_{i} \leq V_{t i \omega} \leq \bar{V}_{i}, \forall t, i, \omega
\end{aligned}
$$




$$
\begin{aligned}
& -\pi \leq \delta_{t i \omega} \leq \pi, \forall t, i, \omega \\
& \lambda_{t I}^{D A}, P_{t}^{D D A} \in \arg (1)^{D A} \\
& \lambda_{t I \omega}^{R T}, P_{t \omega}^{D R T} \in \arg (2)_{\omega}^{R T}
\end{aligned}
$$

where $\Xi^{P D I S C O}=\left\{\lambda_{t}^{D D A}, \lambda_{t \omega}^{D R T}, P_{t k}^{D E R 0}, Q_{t k}^{D E R 0}, P_{t l \omega}^{S D}, Q_{t l \omega}^{S D}, Q_{t i}^{C 0}, P_{t, i j}^{F D 0}, Q_{t, i j}^{F D 0}\right.$, $\left.\delta_{t i}^{0}, V_{t i}^{0}, Q_{t i \omega}^{C}, P_{t, i j, \omega}^{F D}, Q_{t, i j, \omega}^{F D}, \delta_{t i \omega}, V_{t i \omega}\right\}$ is the variable set of the UL PDISCO problem (3). $\Xi^{D U A L}$ is the set of the dual variables.

To minimize the PDISCO's minus-profit (maximize profit), the objective (3.1) of the UL problem is made up of day-ahead and real-time aspects. The dayahead aspect include the revenue/cost from the PDISCO's strategic offers/bids in the day-ahead market, the procurements from the individual DERs, and the revenue of electricity sales to the DL demands. The real-time aspect is the expected minus-profit according to the revenue/cost from the PDISCO's strategic offers/bids in the real-time markets, the penalty of possible DL loadshedding, and the revenue/cost from the DERs' production deviations. The PDISCO's main substation located at the TL bus $I$ is seen as the DL reference bus 1. AC power flow is used to formulate the PDISCO's operation model.

For the day-ahead aspect, at each time $t$ : Constraints (3.2) illustrate the upper bound of the PDISCO's day-ahead offering/bidding quantity. Constraints (3.3) and (3.4) enforce the $\mathrm{AC}$ power balance at the DL reference bus, which keeps the voltage magnitude and voltage angle as constant values with constraints (3.5) and (3.6). Constraints (3.7) impose the capacity limits of the main substation. Constraints (3.8) and (3.9) represent the AC power balance at the other buses. Constraints (3.10) and (3.11) depict the AC power flow through feeder $i-j$, which is restricted by constraints (3.12). Constraints (3.13) specify the production limits of DER $k$. The capacity of each compensator is bounded in constraints (3.14). Constraints (3.15) and (3.16) limit the voltage magnitude and angle at each DL bus.

285 For the real-time aspect, at each time $t$ per scenario $\omega$ : Constraints (3.17) indicate the upper bound of the PDISCO's real-time offering/bidding quantity. Constraints (3.18) and (3.19) guarantee the AC power balance at the DL 
reference bus. Constraints (3.20), (3.21), (3.32) and (3.33) enforce the value of voltage magnitude and angle at each bus $n$. The capacity limits of the main substation, each feeder and each compensator are constrained by (3.22), (3.27) and (3.31), respectively. Constraints (3.23) and (3.24) realize the AC power balance for the other DL buses. The AC power flow is expressed in constraints (3.25) and (3.26). Constraints (3.28)-(3.30) keep the demand power factor constant if DL load-shedding occurs. Note that the PDISCO's offering/bidding prices $\lambda_{t}^{D D A}$ and $\lambda_{t \omega}^{D R T}$ are UL decision variables treated as parameters in the LL problems. Thus, constraints (3.24) and (3.35) represent the interactive impacts between the PDSICO's trading strategies (offering/bidding price and quantity) and markets' outcomes.

\section{4. $M P E C$}

Note that the proposed bilevel model is put forward by the non-linear UL problem and the linear LL problems. Thus, this bilevel model can be translated into a single-level model by replacing the LL markets' problems with their firstorder optimality conditions, and this renders an MPEC. Since the primal-dual approach is more tractable and efficient than the Karush-Kuhn-Tucker (KKT) conditions for off-the-shell branch-and-cut software [5, the former approach is applied in this paper.

The primal-dual approach renders a mathematical program with primal and dual constraints (MPPDC), which is carried out on the transformation of each LL problem. The MPPDCs for the day-ahead market problem and real-time market problems are indicated in (4) and (5), respectively. Constraints (4.1)(4.5) are the dual constraints of the primal constraints (1.2)-(1.11). Constraint (4.6) is the associated strong duality equality of problem (1). For each scenario $\omega$, constraints (5.1)-(5.5) are the dual constraints of the primal constraints (2.2)(2.10), and constraint (5.6) is the associated strong duality equality of problem

315 (2). The strong duality constraint ensures the equality of the primal and dual objective values, one per LL market problem. 
For the LL day-ahead market problem:

$$
\begin{aligned}
& C_{g}^{G}+\lambda_{t n}^{D A}+\gamma_{t g}^{0+}-\gamma_{t g}^{0-}-\eta_{t g}^{0}=0, \forall t, g_{(g: n) \in \mathcal{M}_{G}} \\
& C_{g}^{U P}+\gamma_{t g}^{0+}-\gamma_{t g}^{0-}+\phi_{t g}^{0+}-\phi_{t g}^{0-}=0, \forall t, g \\
& C_{g}^{D N}+\eta_{t g}^{0}+\psi_{t g}^{0+}-\psi_{t g}^{0-}=0, \forall t, g \\
& \lambda_{t}^{D D A}+\left(\lambda_{t I}^{D A}\right)_{n=I}+\beta_{t}^{0+}-\beta_{t}^{0-}=0, \forall t \\
& \quad \sum_{n m \in \Lambda^{T S}} B_{n m}\left(\lambda_{t n}^{D A}-\lambda_{t m}^{D A}\right)+\sigma_{t n}^{0+}-\sigma_{t n}^{0-} \\
& +\sum_{n m \in \Lambda^{T S}} B_{n m}\left(\mu_{t, n m}^{0+}-\mu_{t, m n}^{0+}\right)+\left(\zeta_{t}^{0}\right)_{n=1} \\
& -\sum_{n m \in \Lambda^{T S}} B_{n m}\left(\mu_{t, n m}^{0-}-\mu_{t, m n}^{0-}\right)=0, \forall t, n \\
& \sum_{t, g}\left(C_{g}^{G} P_{t g}^{G}+C_{g}^{U P} R_{t g}^{U P}+C_{g}^{D N} R_{t g}^{D N}\right)+\sum_{t} \lambda_{t}^{D D A} P_{t}^{D D A} \\
& +\sum_{t} \bar{P}^{D S}\left(\beta_{t}^{0+}+\beta_{t}^{0-}\right)+\sum_{t, g} \gamma_{t g}^{0+} \bar{P}_{t g}^{G}+\sum_{t, g} \phi_{t g}^{0+} \bar{R}_{g}^{U P} \\
& +\sum_{t, n m \in \Lambda^{T S}} \bar{P}_{n m}^{T S}\left(\mu_{t, n m}^{0+}+\mu_{t, n m}^{0-}\right)+\sum_{t, n} \lambda_{t n}^{D A} P_{t(d: n) \in \mathcal{M}_{D}}^{T S D} \\
& +\sum_{t, g} \psi_{t g}^{0+} \bar{R}_{g}^{D N}+\sum_{t, n} \pi\left(\sigma_{t n}^{0+}+\sigma_{t n}^{0-}\right)=0
\end{aligned}
$$

For the LL real-time market problems:

$$
\begin{aligned}
& c_{g}^{U P}+\lambda_{t n \omega}^{R T}+\phi_{t g \omega}^{+}-\phi_{t g \omega}^{-}=0, \forall t, g_{(g: n) \in \mathcal{M}_{G}}, \omega \\
& c_{g}^{D N}-\lambda_{t n \omega}^{R T}+\psi_{t g \omega}^{+}-\psi_{t g \omega}^{-}=0, \forall t, g_{(g: n) \in \mathcal{M}_{G}}, \omega \\
& \lambda_{t \omega}^{D R T}+\left(\lambda_{t I \omega}^{R T}\right)_{n=I}+\beta_{t \omega}^{+}-\beta_{t \omega}^{-}=0, \forall t, \omega \\
& \lambda_{t}^{S T}+\lambda_{t(n: d) \in \mathcal{M}_{D} \omega}^{R T}+\nu_{t d \omega}^{+}-\nu_{t d \omega}^{-}=0, \forall t, d, \omega \\
& \sum_{n m \in \Lambda^{T S}} B_{n m}\left(\lambda_{t n \omega}^{R T}-\lambda_{t m \omega}^{R T}\right)+\sigma_{t n \omega}^{+}-\sigma_{t n \omega}^{-} \\
& +\sum_{n m \in \Lambda^{T S}} B_{n m}\left(\mu_{t, n m, \omega}^{+}-\mu_{t, m n, \omega}^{+}\right)+\left(\zeta_{t \omega}\right)_{n=1} \\
& -\sum_{n m \in \Lambda^{T S}} B_{n m}\left(\mu_{t, n m, \omega}^{-}-\mu_{t, m n, \omega}^{-}\right)=0, \forall t, n, \omega \\
& \sum_{t, g}\left(c_{g}^{U P} r_{t g \omega}^{U P}+c_{g}^{D N} r_{t g \omega}^{D N}\right)+\sum_{t} \lambda_{t \omega}^{D R T} P_{t \omega}^{D R T}
\end{aligned}
$$




$$
\begin{aligned}
& +\sum_{t} \bar{P}^{D S}\left(\beta_{t \omega}^{+}+\beta_{t \omega}^{-}\right)+\sum_{t, d} \lambda_{t}^{S T} P_{t d \omega}^{S T} \\
& +\sum_{t, g} \phi_{t g \omega}^{+} R_{t g}^{U P}+\sum_{t, g} \psi_{t g \omega}^{+} R_{t g}^{D N}+\sum_{t, n} \pi\left(\sigma_{t n \omega}^{+}+\sigma_{t n \omega}^{-}\right) \\
& +\sum_{t, d} \nu_{t d \omega}^{+} P_{t d}^{T S D}-\sum_{t, n} \lambda_{n \omega}^{R T} \sum_{n m \in \Lambda^{T S}} B_{n m}\left(\theta_{t n}^{0}-\theta_{t m}^{0}\right) \\
& +\sum_{n m \in \Lambda^{T S}} \bar{P}_{t, n m}^{T S}\left(\mu_{t, n m, \omega}^{+}+\mu_{t, n m, \omega}^{-}\right)=0, \forall \omega
\end{aligned}
$$

Substituting each LL market problem with the corresponding MPPDC, the proposed bilevel model is transformed into a single-level optimization problem, as expressed in (6). Thus, the commercial off-the-shelf large-scale non-linear optimization solver CONOPT3 [23] can be employed to solve this reformulated non-linear model.

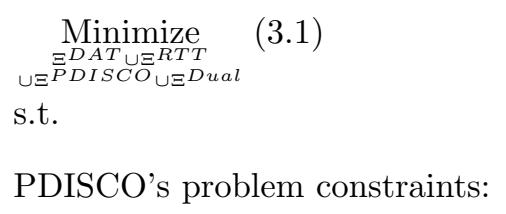

$(3.2)-(3.35)$

Day-ahead market problem MPPDC constraints:

$(1.2)-(1.11)$ and $(4)$;

Real-time market problems MPPDC constraints:

$(2.2)-(2.10)$ and $(5)$.

\section{Case Studies}

In this section, a modified 14-bus DL network 24] interconnected to a 9-bus TL network [25] is used to validate the effectiveness of the proposed bilevel model for deriving the PDISCO's strategic offers/bids and markets' performances.

To simulate the uncertainties of individual DERs, the scenario handling approach [4] is adopted to create 1000 scenarios and reduce to 15 scenarios for the case studies. 
All cases are implemented on a $3.6 \mathrm{GHz}$ Intel Core i7 processor based on a with GAMS 24.4.1 23 .

\subsection{Data}

For the PDISCO-owned 14-bus network: The capacities of the main substation $\bar{S}$ and each feeder $\bar{S}_{i j}$ are set to 200 MVA and 100 MVA, respectively.

to 1.1 p.u.. The transformer tap ratio $\tau_{i}$ is imposed to 1 . Each compensator is with 0-80 MVar. The WTs and PVs are selected to represent the stochastic DERs. The mappings of the DERs and buses are WT1:11, WT2:13, WT3:9, PV1:2, PV2:7, and PV3:12. For simplicity, the capacity limit of each DER is The PDISCO rationally procures the DERs' portfolios and strategically trades in individual markets with 24 times per stage. The purchase price $\lambda_{t}^{D E R}$ for each DER's generation is assumed to be unique, as shown in Table I, which also includes the sales price $\lambda_{t}^{D S D}$, and network operation cost $C_{t}^{D S}$. The DL multi${ }_{345}$ plier $\alpha_{t}$ is used to obtain the demand $P_{t l}^{D S D}, Q_{t l}^{D S D}$ on the base value described in 24 .

For the market-operated 9-bus network: The PDISCO's network is assumed to be interconnected at TL bus 4 with a power injection limit $100 \mathrm{MW}$. The line capacity is set to $500 \mathrm{MW}$. Table II shows the capacity, reserve limit and corresponding cost of each generator. The real-time regulation $\operatorname{cost} c_{g}^{U P}$ and $c_{g}^{D N}$ can be regarded as the generation cost $C_{g}^{G}$. The TL multiplier $\beta_{t}$ shown in Table I is used to get the day-ahead bidding price $\lambda_{t d}^{T S D}$ relying on the base value $45 € / \mathrm{MW}$ and consumption $P_{t d}^{T S D}$ according to the base in [25]. In addition, the DL and TL load-shedding prices $\left(\lambda_{t}^{S D}, \lambda_{t}^{S T}\right)$ are considered as 200 times as ${ }_{355} \lambda_{t}^{D D A}$ and $\lambda_{t \omega}^{D R T}$, respectively.

Other DL and TL parameters can be found in [24] 25]. All the price parameters mentioned above are estimated by the NordPool [26] prices. 
Table 1: Essential Input Parameters for the PDISCO Tading in Markets

\begin{tabular}{|c|c|c|c|c|c|}
\hline $\begin{array}{c}t \\
{[\text { Hour] }}\end{array}$ & $\begin{array}{c}\lambda_{t}^{D E R} \\
{[€]}\end{array}$ & $\begin{array}{c}\lambda_{t}^{D S D} \\
{[€]}\end{array}$ & $\begin{array}{c}C_{t}^{D S} \\
{[€]}\end{array}$ & $\alpha_{t}$ & $\beta_{t}$ \\
\hline 1 & 1.0 & 12.0 & 1.6 & 4.0 & 0.3 \\
\hline 2 & 1.9 & 22.8 & 3.0 & 7.6 & 0.3 \\
\hline 3 & 2.0 & 24.0 & 3.2 & 8.0 & 0.3 \\
\hline 4 & 2.5 & 30.0 & 4.0 & 10.0 & 0.3 \\
\hline 5 & 3.5 & 42.0 & 5.6 & 14.0 & 0.8 \\
\hline 6 & 4.3 & 51.0 & 6.8 & 17.0 & 0.8 \\
\hline 7 & 5.0 & 60.0 & 8.0 & 20.0 & 0.9 \\
\hline 8 & 5.5 & 66.0 & 8.8 & 22.0 & 0.9 \\
\hline 9 & 5.8 & 69.0 & 9.2 & 23.0 & 1.0 \\
\hline 10 & 8.0 & 96.0 & 12.8 & 32.0 & 1.7 \\
\hline 11 & 9.0 & 108.0 & 14.4 & 36.0 & 1.8 \\
\hline 12 & 9.6 & 115.2 & 15.4 & 38.4 & 1.8 \\
\hline 13 & 9.0 & 108.0 & 14.4 & 36.0 & 1.7 \\
\hline 14 & 5.5 & 66.0 & 8.8 & 22.0 & 1.0 \\
\hline 15 & 4.5 & 54.0 & 7.2 & 18.0 & 1.0 \\
\hline 16 & 4.8 & 57.6 & 7.7 & 19.2 & 1.0 \\
\hline 17 & 5.5 & 66.0 & 8.8 & 22.0 & 1.0 \\
\hline 18 & 8.3 & 99.0 & 13.2 & 33.0 & 1.5 \\
\hline 19 & 8.5 & 102.0 & 13.6 & 34.0 & 1.6 \\
\hline 20 & 9.0 & 108.0 & 14.4 & 36.0 & 1.7 \\
\hline 21 & 9.5 & 114.0 & 15.2 & 38.0 & 1.8 \\
\hline 22 & 3.5 & 42.0 & 5.6 & 14.0 & 0.8 \\
\hline 23 & 2.1 & 25.2 & 3.4 & 8.4 & 0.5 \\
\hline 24 & 1.5 & 18.0 & 2.4 & 6.0 & 0.4 \\
\hline
\end{tabular}

Table 2: Input Parameters for Generators

\begin{tabular}{c|c|c|c|c|c|c}
\hline No. & $\begin{array}{c}\bar{P}_{g}^{G} \\
{[\mathrm{MW}]}\end{array}$ & $\begin{array}{c}C_{g}^{G} \\
{[€ / \mathrm{MW}]}\end{array}$ & $\begin{array}{c}C_{g}^{U P} \\
{[€ / \mathrm{MW}]}\end{array}$ & $\begin{array}{c}C_{g}^{D N} \\
{[€ / \mathrm{MW}]}\end{array}$ & $\begin{array}{c}\bar{R}_{g}^{U P} \\
{[\mathrm{MW}]}\end{array}$ & $\begin{array}{c}\bar{R}_{g}^{D N} \\
{[\mathrm{MW}]}\end{array}$ \\
\hline 1 & 1500 & 27 & 30 & 22 & 500 & 500 \\
\hline 2 & 1200 & 29 & 32 & 24 & 300 & 300 \\
\hline 3 & 1200 & 36 & 38 & 30 & 300 & 300 \\
\hline
\end{tabular}




\subsection{PDISCO trading strategies}

The PDISCO's strategic offers and bids make up continuous curves for trad-

significantly during hours 1-4 and 23-24. Consequently, the PDISCO's profits by trading in markets are obtained and shown in Fig. 4. Scenario-based $(\omega=1)$ real-time profits are recorded for comparison with the day-ahead profits. Ob- 


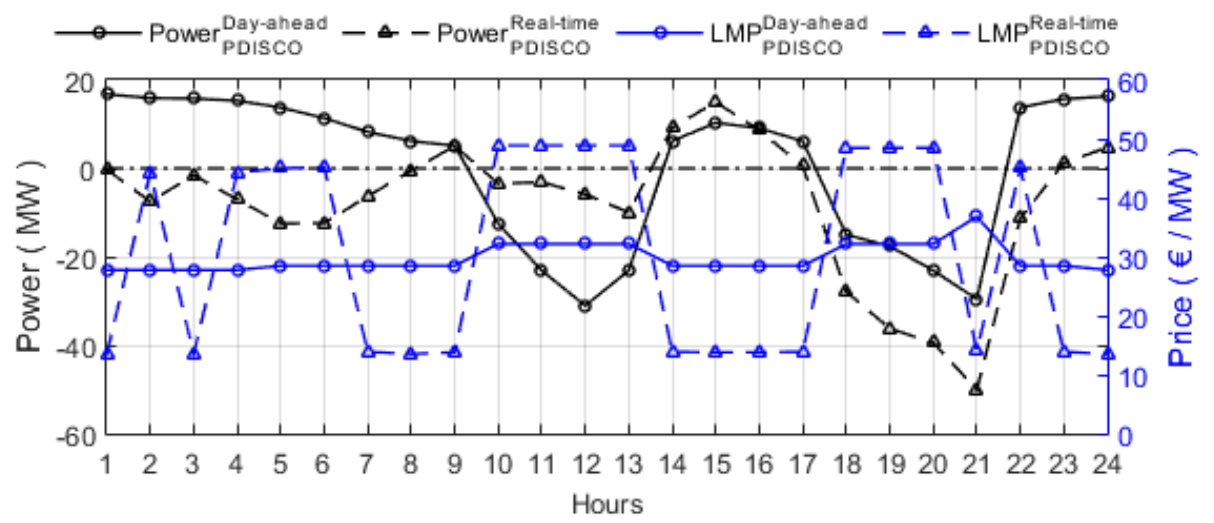

Figure 2: The PDISCO's strategic offers and bids in two-stage markets.

serve that, the day-ahead profit peaks occur consistently with the load peaks. 


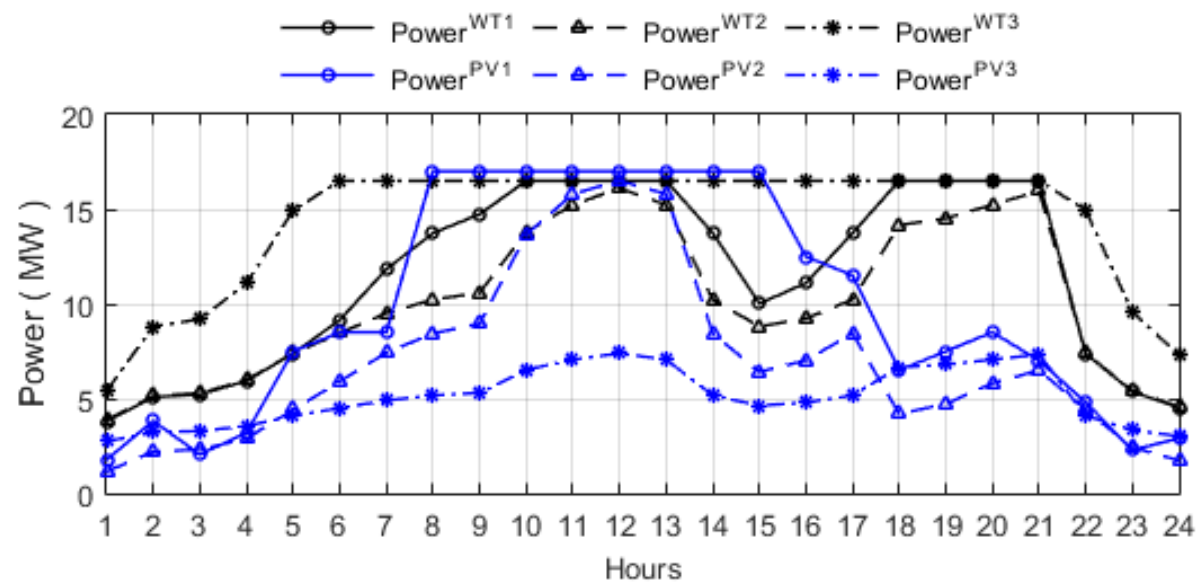

Figure 3: The PDISCO's power procurements from individual DERs.

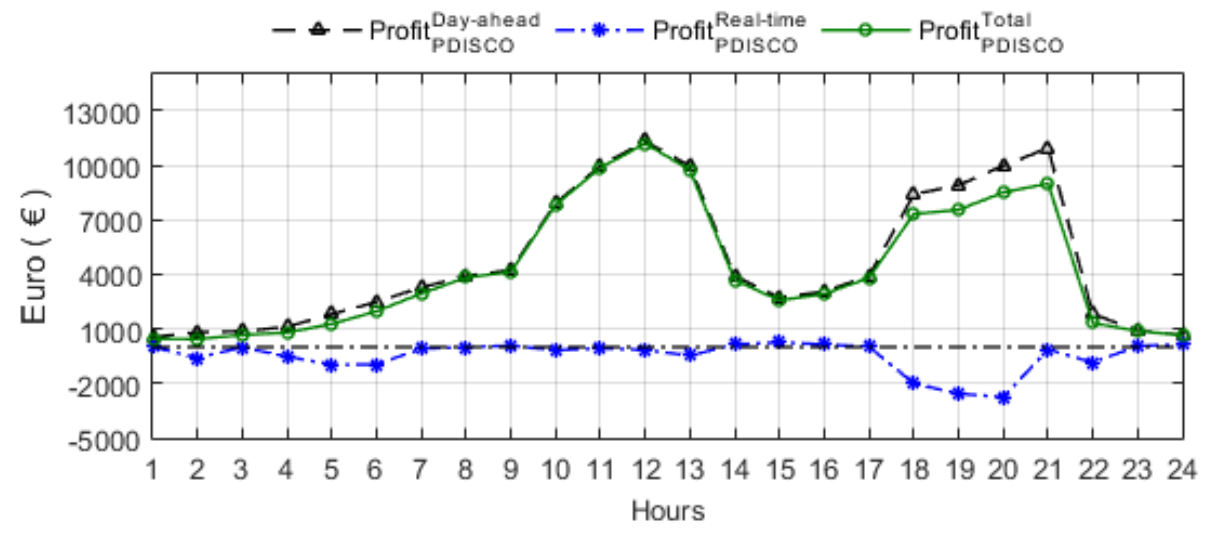

Figure 4: The PDISCO's day-ahead, real-time and total profits. 
generation drop for generator 1 on day-ahead offers, in total $19 \%$ per day. In order to mitigate the unpredictable oscillations of the PDISCO's offers/bids, the notable power increment occurs in both reserve and regulation circumstances, i.e., $192 \%$ (71\%) and 553\% (56\%) for daily upward (downward) reserve and up (down) regulation, respectively. The peaks of the upward reserve and up regulation capacity are coincidentally launched at hour 21, corresponding to the PDISCO's heavy arbitrage scheme indicated above as for Fig. 2 and 4 . The effect of the PDISCO's moderate arbitrage at hour 7 is not explicitly expressed, since the upward reserve and up regulation power of the generator 1 are already merged in the system-wide reserve and regulation capacity, respectively.

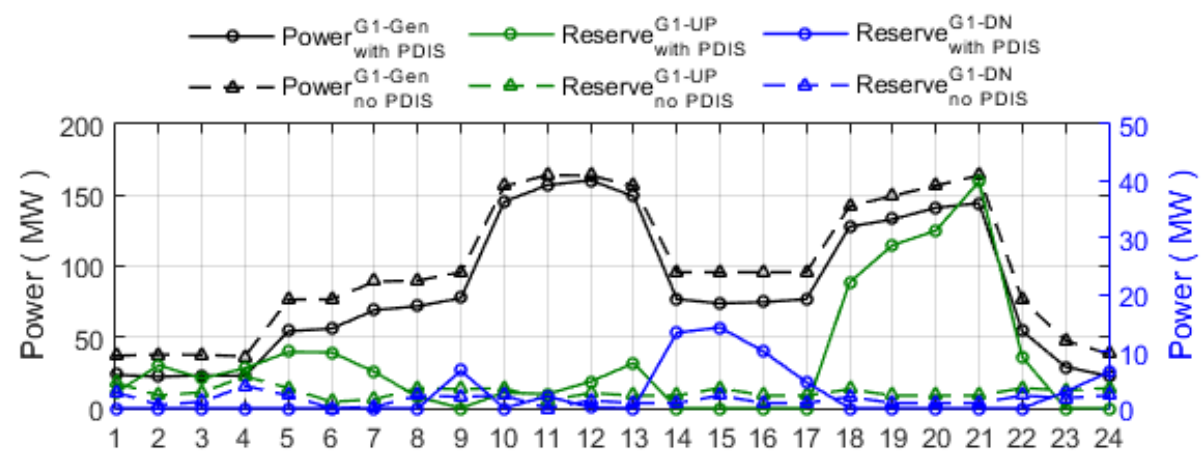

( a )

Hours

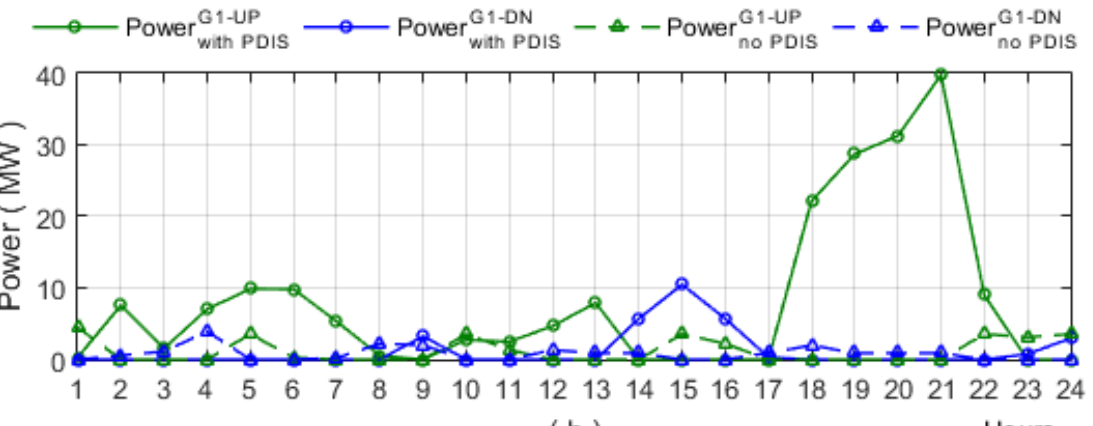

(b)

Hours

Figure 5: Cleared power quantities from markets for generator 1. 


\subsection{Impact on TL markets}

In addition, the comparison results also include the day-ahead social welfare and real-time operation cost of the markets, as shown in Fig. 6. The social welfare continuously declines by considering the PDISCO's participation, reducing by $12 \%$ daily. In contrast, the cost increases by $962 \%$ for the real-time market operation. In other words, the PDISCO's strategic trading negatively impacts the markets' objectives.

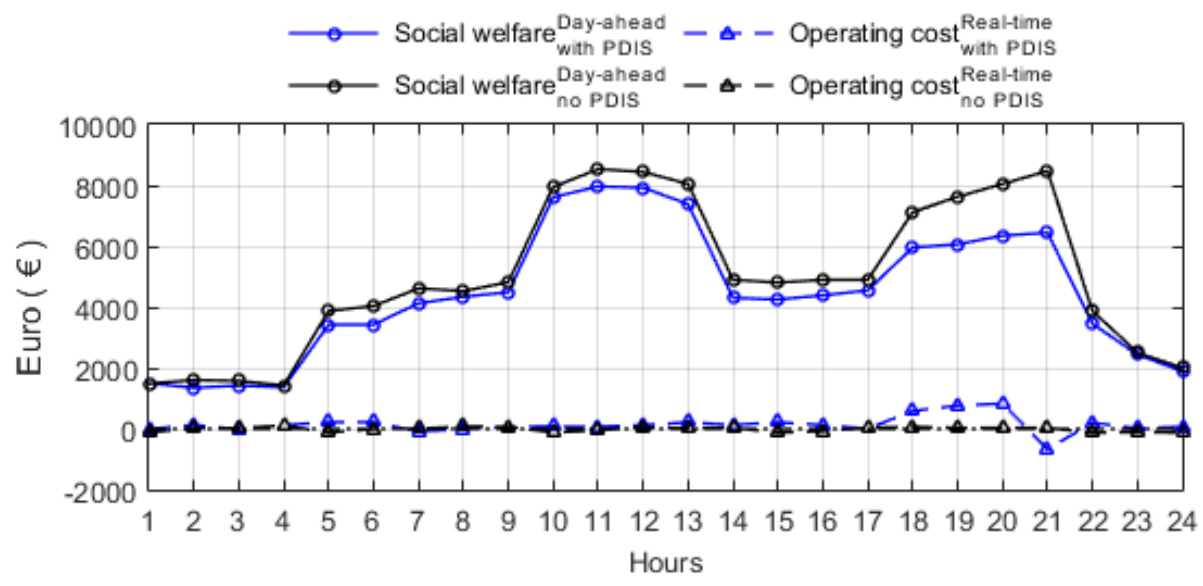

Figure 6: Day-ahead social welfare and real-time operation cost of markets.

Concerning the PDISCO's trading strategies essentially depend on the DERs' by resetting the DERs' availability in additional cases. Thus, the discussions above are listed as Case 1. Case 2 increases the individual DERs' capacities to two-times higher than Case 1, while these are reduced to half in Case 3. The results of PDISCO's profits and markets' objectives are summarized in Table III. The obtained social welfare is almost the same in Cases 1 and 3, but falls by $13 \%$ for Case 2 . This means higher penetration of DERs yields more offers from the PDISCO and lower social welfare in the day-ahead market. The operation cost of the real-time market increases significantly with the DERs' capacity growth in Cases 1-3. Observe that the higher DERs can not ensure a 
higher profit for the PDISCO, while lower DERs definitely bring lower profit, and even render minus-profit (in Case 3). To improve the profitability for the PDISCO, appropriate portfolio procurement is the best option.

\begin{tabular}{c|c|c|c}
\multicolumn{4}{c}{ Table 3: Achievements of the PDISCO and Markets } \\
\hline No. & $\begin{array}{c}\text { PDISCO } \\
\text { profit }[€]\end{array}$ & $\begin{array}{c}\text { Day-ahead market } \\
\text { social welfare }[€]\end{array}$ & $\begin{array}{c}\text { Real-time market } \\
\text { cost }(\omega=1)[€]\end{array}$ \\
\hline Case 1 & 11334.8 & 10642.9 & 339.5 \\
\hline Case 2 & 10457.5 & 9300.8 & 1497.9 \\
\hline Case 3 & -54138.2 & 10654.4 & 237.0 \\
\hline
\end{tabular}

\section{Conclusion}

This paper presents a bilevel model for a PDISCO strategically trading ology can assist the PDISCO to seek the appropriate portfolio procurements from DERs in differing production scenarios. 
3) A PDISCO can be seen as a centralized renewable energy source (e.g., large-scale wind or PV farms), which can be considered to integrate in the wholesale markets. This may facilitate the DL purchases of DERs to interact with the TL markets in real life. Besides, the DL trading between DERs and the PDISCO can be extended to multiple mechanisms with various clearing procedures, such as ancillary market, reserve market, and flexibility market, which could enhance the energy efficiency and system reliability.

4) As the interface actor, the PDISCO's procurement with DL DERs and trading in the TL markets are exhibited as hierarchical structures, which can be formulated by a tri-level model. The corresponding modeling and solving approaches can be investigated in the future.

\section{Acknowledgements}

The authors would like to acknowledge the support from the Research Council of Norway under Grant 255209, the Danish iPower Platform Project under Grant 10-095378, and the U.S. Department of Energy (DOE)'s Office of Electricity Delivery and Energy Reliability.

\section{References}

475 [1] Q. Wang, C. Zhang, Y. Ding, G. Xydis, J. Wang, J. Østergaard, Review of real-time electricity markets for integrating distributed energy resources

口 and demand response, Appl. Energy 138 (2015) 695-706. doi:10.1016/j. apenergy.2014.10.048.

[2] P. Sorknæs, H. Lund, A. N. Andersen, Future power market and sustainable energy solutions - The treatment of uncertainties in the daily operation of combined heat and power plants, Appl. Energy 144 (2015) 129-138. doi:10.1016/j.apenergy.2015.02.041.

[3] NewYorkState, Reforming the energy vision, NYS Department of Public Service. 
[4] Z. Wang, B. Chen, J. Wang, M. M. Begovic, C. Chen, Coordinated energy management of networked microgrids in distribution systems, IEEE Trans. Smart Grid 6 (1) (2015) 45-53. doi:10.1109/TSG.2014.2329846

[5] S. A. Gabriel, A. J. Conejo, J. D. Fuller, B. F. Hobbs, C. Ruiz, Complementarity modeling in energy markets, Springer, New York, 2012.

[6] H. Haghighat, S. W. Kennedy, A bilevel approach to operational decision making of a distribution company in competitive environments, IEEE Trans. on Power Syst. 27 (4) (2012) 1797-1807. doi:10.1109/TPWRS. 2011. 2182214

[7] R. Palma-Behnke, S. Member, J. L. C. A, L. S. Vargas, A distribution company energy acquisition market model with integration of distributed generation and load curtailment options, IEEE Trans. Power Syst. 20 (4) (2005) 1718-1727. doi:10.1109/TPWRS.2005.857284.

[8] H. Li, Y. Li, Z. Li, A multiperiod energy acquisition model for a distribution company with distributed generation and interruptible load, IEEE Trans. Power Syst. 22 (2) (2007) 588-596. doi:10.1109/TPWRS.2007.894862.

[9] C. Zhang, Q. Wang, J. Wang, P. Pinson, J. M. Morales, J. Østergaard, Real-time procurement strategies of a proactive distribution company with aggregator-based demand response, IEEE Trans. Smart Grid (2016, in press) $1-10$.

[10] C. Zhang, Market design and strategy making for proactive distribution grid with DERs, Technical University of Denmark, Denmark, 2015.

[11] J. Wang, M. Shahidehpour, Z. Li, A. Botterud, Strategic generation capacity expansion planning with incomplete information, IEEE Trans. Power Syst. 24 (2) (2009) 1002-1010. doi:10.1109/TPWRS.2009.2017435.

[12] L. Baringo, A. J. Conejo, Strategic offering for a wind power producer, IEEE Trans. on Power Syst. 28 (4) (2013) 4645-4654. doi:10.1109/TPWRS. 2013.2273276 . 
[13] R. Fernández-Blanco, J. M. Arroyo, N. Alguacil, Network-constrained dayahead auction for consumer payment minimization, IEEE Trans. on Power Syst. 99 (2) (2013) 1-11. doi:10.1109/TPWRS.2013.2284283

[14] A. B. Makler, S. S. Schleimer, Preserving the Benefits of Competition through Effective Competitive Bidding Rules for Utility Resource Procurement, The Electricity Journal 16 (6) (2003) 27-36. doi:10.1016/ S1040-6190(03) 00078-2.

[15] D. T. Nguyen, L. B. Le, Optimal bidding strategy for microgrids considering renewable energy and building thermal dynamics, IEEE Transactions on Smart Grid 5 (4) (2014) 1608-1620. doi:10.1109/TSG.2014.2313612.

[16] W. Tian, Z. Li, M. Shahidehpour, Renewable energy procurement in Illinois, Electricity Journal 27 (2) (2014) 43-51. doi:10.1016/j.tej.2014. 02.003

[17] G. Díaz, B. Moreno, J. Coto, J. Gómez-Aleixandre, Valuation of wind power distributed generation by using Longstaff-Schwartz option pricing

(1) method, Appl. Energy 145 (2015) 223-233. doi:10.1016/j.apenergy. 2015.02 .046

[18] P. Siano, D. Sarno, Assessing the benefits of residential demand response in a real time distribution energy market, Appl. Energy 161 (2016) 533-551. doi:10.1016/j.apenergy.2015.10.017.

[19] R. Arghandeh, J. Woyak, A. Onen, J. Jung, R. P. Broadwater, Economic optimal operation of Community Energy Storage systems in competitive en535 ㅁ ergy markets, Appl. Energy 135 (2014) 71-80. doi:10.1016/j.apenergy. 2014.08 .066

[20] Z. Li, Q. Guo, H. Sun, J. Wang, Storage-like devices in load leveling: Complementarity constraints and a new and exact relaxation method, Appl. Energy 151 (2015) 13-22. doi:10.1016/j.apenergy.2015.04.061. 
[21] N. Zhang, Y. Yan, W. Su, A game-theoretic economic operation of residential distribution system with high participation of distributed electricity 2015.05 .011

[22] J. Zhong, L. He, C. Li, Y. Cao, J. Wang, B. Fang, L. Zeng, G. Xiao, Coordinated control for large-scale EV charging facilities and energy storage devices participating in frequency regulation, Appl. Energy 123 (2014) 253-262. doi:10.1016/j.apenergy.2014.02.074.

[23] GAMS, [Online]. Available: http://www.gams.com/.

[24] F. R. Alonso, D. Q. Oliveira, a. C. Zambroni de Souza, Artificial im550 mune systems optimization approach for multiobjective distribution system reconfiguration, IEEE Trans. on Power Syst. 30 (2) (2015) 840-847. doi:10.1109/TPWRS.2014.2330628

[25] A. Jamehbozorg, G. Radman, Small signal analysis of power systems with wind and energy storage units, IEEE Trans. on Power Syst. 30 (1) (2015) 555 298-305. doi:10.1109/TPWRS.2014.2321711.

[26] Nordpool, [Online]. Available: http://www.nordpoolspot.com/. 